\title{
Singular integral operators with non-smooth kernels on irregular domains
}

Xuan Thinh Duong and Alan McIntosh

Abstract. Let $\mathcal{X}$ be a space of homogeneous type. The aims of this paper are as follows.

i) Assuming that $T$ is a bounded linear operator on $L_{2}(\mathcal{X})$, we give a sufficient condition on the kernel of $T$ so that $T$ is of weak type $(1,1)$, hence bounded on $L_{p}(\mathcal{X})$ for $1<p \leq 2$; our condition is weaker than the usual Hörmander integral condition.

ii) Assuming that $T$ is a bounded linear operator on $L_{2}(\Omega)$ where $\Omega$ is a measurable subset of $\mathcal{X}$, we give a sufficient condition on the kernel of $T$ so that $T$ is of weak type $(1,1)$, hence bounded on $L_{p}(\Omega)$ for $1<p \leq 2$.

iii) We establish sufficient conditions for the maximal truncated operator $T_{*}$, which is defined by $T_{*} u(x)=\sup _{\varepsilon>0}\left|T_{\varepsilon} u(x)\right|$, to be $L_{p}$ bounded, $1<p<\infty$. Applications include weak $(1,1)$ estimates of certain Riesz transforms, and $L_{p}$ boundedness of holomorphic functional calculi of linear elliptic operators on irregular domains.

\section{Introduction.}

Let $(\mathcal{X}, d, \mu)$ be a space of homogeneous type, equipped with a metric $d$ and a measure $\mu$. Let $T$ be a bounded linear operator on 
$L_{2}(\mathcal{X})$ with an associated kernel $k(x, y)$ in the sense that

$$
(T f)(x)=\int_{\mathcal{X}} k(x, y) f(y) d \mu(y)
$$

where $k(x, y)$ is a measurable function, and the above formula holds for each continuous function $f$ with compact support, and for almost all $x$ not in the support of $f$.

One important result of Calderón-Zygmund operator theory is the well known Hörmander integral condition on the kernel $k(x, y)$, see [Hör], which is a sufficient condition for the operator $T$ to be of weak type $(1,1)$. It states that $T$ satisfies weak $(1,1)$ estimates if there exist constants $C$ and $\delta>1$ so that

$$
\int_{d(x, y) \geq \delta d\left(y_{1}, y\right)}\left|k(x, y)-k\left(x, y_{1}\right)\right| d \mu(x) \leq C,
$$

for all $y, y_{1} \in \mathcal{X}$.

In practice, many operators satisfy the Hörmander integral condition, but there are numerous examples of operators which do not, and certain classes of such operators can be proved to be of weak type $(1,1)$. See, for example $[\mathrm{F}]$, [Ch1], [CR], [Hof], [Se]. However, in these papers, the authors investigate specific classes of operators and do not give sufficient conditions on kernels for general operators to be of weak type $(1,1)$.

A natural question is whether one can weaken the Hörmander integral condition and still conclude that $T$ is of weak type $(1,1)$. Although Calderón-Zygmund operator theory is now well established, to our best knowledge, no such condition is known. Our first aim is to give a positive answer to this open question.

There is another limitation of the usual Calderón-Zygmund theory. It is only established for spaces of homogeneous type. The main feature of these spaces is that they satisfy the doubling property. Measurable subsets of $\mathbb{R}^{n}$ which do not possess any smoothness of their boundaries, do not satisfy the doubling property, hence they are not spaces of homogeneous type. Such measurable sets, however, do appear naturally in partial differential equations. Our second aim is to present a sufficient condition on the kernel of a bounded operator $T$ on $L_{2}(\Omega)$, where $\Omega$ is a measurable subset of a space of homogeneous type, so that $T$ is of weak type $(1,1)$ on $\Omega$.

The paper is organised as follows. In Section 2, we assume that $T$ is a bounded linear operator on $L_{2}(\mathcal{X})$, where $\mathcal{X}$ is a space of homogeneous 
type. We then prove a sufficient condition on the kernel $k(x, y)$ of $T$ so that $T$ is of weak type $(1,1)$ (Theorem 1 ). Roughly speaking, $T$ is of weak type $(1,1)$ if there exists a class of operators $A_{t}$ with kernels $a_{t}(x, y)$, which play the role of approximations to the identity, so that the kernels $k_{t}(x, y)$ of the composite operators $T A_{t}$ satisfy the condition

$$
\int_{d(x, y) \geq c t^{1 / m}}\left|k(x, y)-k_{t}(x, y)\right| d \mu(x) \leq C,
$$

for some positive constants $m, c, C$, uniformly in $y \in \mathcal{X}$ and $t>0$. The freedom in choosing $A_{t}$ is important. In particular circumstances we may require them to commute with $T$, or we may wish to allow the kernels $a_{t}$ to be discontinuous.

It is not difficult to check that our condition is a consequence of the Hörmander integral condition (Proposition 1).

In Section 3, we assume that $\Omega$ is a measurable subset of a space of homogeneous type with no smoothness on the boundary. We then present a sufficient condition on the kernel $k(x, y)$ which is somewhat stronger than that of Theorem 1, so that the operator $T$ is of weak type $(1,1)$ on $\Omega$ (Theorem 2 ). Our result gives new criteria to investigate the $L_{p}$ boundedness of singular integrals on measurable sets. The results on $\Omega$ are made possible by the fact that no smoothness is required on the kernels $a_{t}(x, y)$ in Theorem 1 .

In Section 4, we extend the results in sections 2 and 3 to establish sufficient conditions on the kernel $k(x, y)$ which ensure the $L_{p}$ boundedness of the maximal truncated operator $T_{*}$, where $T_{*} u(x)=$ $\sup _{\varepsilon>0}\left|T_{\varepsilon} u(x)\right|$ and

$$
T_{\varepsilon} u(x)=\int_{d(x, y) \geq \varepsilon} k(x, y) u(y) d \mu(y) .
$$

Our assumptions on the kernel $k(x, y)$ are somewhat stronger than those used in Theorems 1 and 2, but are essentially weaker than the usual ones on spaces of homogeneous type (Theorem 3). The result is new for measurable subsets of spaces of homogeneous type (Theorem 4).

Applications are given in Section 5. We first establish weak $(1,1)$ estimates for certain Riesz transforms and similar types of operators (Theorem 5). This allows us, for example, to simplify the proof of the $L_{p}$ boundedness of the Riesz transforms on Lie groups which was given by Saloff-Coste when $1<p \leq 2$ [SC].

Finally, we prove that every operator $L$ with a bounded holomorphic functional calculus in $L_{2}(\Omega)$, which generates a semigroup with 
suitable upper bounds on its heat kernels, also has a bounded holomorphic functional calculus in $L_{p}(\Omega)$ when $1<p<\infty$ (Theorem 6). Here $\Omega$ is a measurable subset of a space $\mathcal{X}$ of homogeneous type. It is this result which prompted our investigation, so let us outline its background.

In the case when the heat kernels also satisfy Hölder bounds, then this result follows from the usual Calderón-Zygmund theory, because the operators $f(L)$ in the functional calculus satisfy standard CalderónZygmund bounds. This is the approach developed by Duong in the case of those elliptic operators having such heat kernels, which are defined by boundary conditions on strongly Lipschitz domains. See his thesis $[\mathrm{Du}]$ and also $\left[\mathrm{DM}^{\mathrm{c}}\right]$. This method does not work for those elliptic operators whose heat kernels satisfy pointwise bounds but not Hölder bounds. In [DR], Duong and Robinson showed how to proceed in such cases, provided still that the operators are defined on strongly Lipschitz domains. There they proved the first part of Theorem 6 of this paper in the case when $\Omega$ is a space of homogeneous type, though the last part, namely the $L_{p}$ boundedness of the maximal truncated operators, is new. In $[\mathrm{AE}]$, Arendt and ter Elst applied this theorem to the Dirichlet problem for certain elliptic operators defined on subsets of $\mathbb{R}^{n}$ whose boundary has null measure, by extending the functional calculus to that of an operator defined on all of $\mathbb{R}^{n}$. They asked whether the assumption concerning the null measure of the boundary could be dropped. This is what we do in Theorem 6 .

As can be seen, our investigations into removing the assumption of Hölder continuity from the kernels have led to the formulation of general conditions on singular integral operators which are applicable in a variety of situations.

\section{Weak $(1,1)$ estimates of singular integral operators.}

Let $\mathcal{X}$ be a topological space equipped with a measure $\mu$ and a metric $d$ which is a measurable function on $\mathcal{X} \times \mathcal{X}$. We define $\mathcal{X}$ to be $a$ space of homogeneous type if the balls $B(x ; r)=\{y \in \mathcal{X}: d(x, y)<r\}$ satisfy the doubling property

$$
\mu(B(x ; 2 r)) \leq c \mu(B(x ; r))<+\infty,
$$

for some $c \geq 1$ uniformly for all $x \in \mathcal{X}$ and $r>0$. A more general definition can be found in [CW, Chapter 3]. 
Note that the doubling property implies the following strong homogeneity property,

$$
\mu(B(x ; \lambda r)) \leq c \lambda^{n} \mu(B(x ; r))
$$

for some $c, n>0$ uniformly for all $\lambda \geq 1$. The parameter $n$ is a measure of the dimension of the space. There also exist $c$ and $N, 0 \leq N \leq n$ so that

$$
\mu(B(y ; r)) \leq c\left(1+\frac{d(x, y)}{r}\right)^{N} \mu(B(x ; r))
$$

uniformly for all $x, y \in \mathcal{X}$ and $r>0$. Indeed, the property (U) with $N=n$ is a direct consequence of triangle inequality of the metric $d$ and the strong homogeneity property. In the cases of Euclidean spaces $\mathbb{R}^{n}$ and Lie groups of polynomial growth, $N$ can be chosen to be 0 .

Let $T$ be a bounded linear operator mapping $L_{2}(\mathcal{X})$ into $L_{2}(\mathcal{X})$. Assume the operator $T$ is given by a kernel $k(x, y)$ in the sense of $(1)$.

We shall work with a class of integral operators $A_{t}, t>0$, which plays the role of approximations to the identity. We assume the operators $A_{t}$ can be represented by kernels $a_{t}(x, y)$ in the sense that

$$
A_{t} u(x)=\int_{\mathcal{X}} a_{t}(x, y) u(y) d \mu(y)
$$

for every function $u \in L_{2}(\mathcal{X}) \cap L_{1}(\mathcal{X})$, and the kernels $a_{t}(x, y)$ satisfy the following conditions

$$
\left|a_{t}(x, y)\right| \leq h_{t}(x, y)
$$

for all $x, y \in \mathcal{X}$ where $h_{t}(x, y)$ is a function satisfying

$$
h_{t}(x, y)=\left(\mu\left(B\left(x ; t^{1 / m}\right)\right)\right)^{-1} s\left(d(x, y)^{m} t^{-1}\right)
$$

in which $m$ is a positive constant and $s$ is a positive, bounded, decreasing function satisfying

$$
\lim _{r \rightarrow \infty} r^{n+\kappa} s\left(r^{m}\right)=0
$$

for some $\kappa>N$, where $N$ is the power which appeared in property (U), and $n$ the "dimension" entering the strong homogeneity property. 
It then follows that

$$
\begin{aligned}
h_{t}(x, y) \leq & c \min \left\{\frac{1}{\mu\left(B\left(x ; t^{1 / m}\right)\right)}, \frac{1}{\mu\left(B\left(y ; t^{1 / m}\right)\right)}\right\} \\
& \cdot\left(1+\frac{d(x, y)}{t^{1 / m}}\right)^{N} s\left(d(x, y)^{m} t^{-1}\right) \\
\leq & c \min \left\{\frac{1}{\mu\left(B\left(x ; t^{1 / m}\right)\right)}, \frac{1}{\mu\left(B\left(y ; t^{1 / m}\right)\right)}\right\} s_{1}\left(d(x, y)^{m} t^{-1}\right),
\end{aligned}
$$

where $s_{1}$ is a function similar to $s$ with some $\kappa>0$.

We also note that there exist positive constants $c_{1}$ and $c_{2}$ so that

$$
c_{1} \leq \int_{\mathcal{X}} h_{t}(x, y) d \mu(x) \leq c_{2}
$$

uniformly in $t$ and $y$.

The existence of such a class of operators $A_{t}$ in a space of homogeneous type, is not a problem. We can first choose a function $s$ satisfying the decay condition in (3), define $h_{t}$ as in (3), and let $a_{t}=h_{t}$, hence conditions (2) and (3) are automatically satisfied. The kernels $a_{t}$ then possess the smoothness of the function $s$.

For any $m>0$, we can also construct $a_{t}(x, y)$ with the following additional properties

$$
\begin{aligned}
a_{t}(x, y)= & 0, \quad \text { when } d(x, y) \geq c_{0} t^{1 / m}, \\
& \int_{\mathcal{X}} a_{t}(x, y) d \mu(x)=1,
\end{aligned}
$$

for all $y \in \mathcal{X}, t>0$. This can be achieved by choosing

$$
a_{t}(x, y)=\left(\mu\left(B\left(y ; t^{1 / m}\right)\right)\right)^{-1} \chi_{B\left(y ; t^{1 / m}\right)}(x),
$$

where $\chi_{B\left(y ; t^{1 / m}\right)}$ denotes the characteristic function on the ball $B\left(y ; t^{1 / m}\right)$. Then let $A_{t}$ be the operators which are given by the kernels $a_{t}(x, y)$.

These operators $A_{t}$ constructed as above exist in the space $\mathcal{X}$ independently of the operator $T$. However, for certain operators $T$, it is useful to construct operators $A_{t}$ which are related to $T$. This is of interest since the analysis of $A_{t}, T A_{t}$ and $A_{t} T$ is useful for establishing 
the boundedness of $T$ in an $L_{p}$ space. Examples of this are given in Section 5 .

The following lemma is needed in the proof of Theorem 1. For its proof, see [DR, Proposition 2.5].

Lemma 1. Given functions $h_{t}(x, z)$ which satisfy $(3)$, and $\nu>0$, there exist positive constants $c$ and $\theta$ such that

$$
\sup _{z \in B(y, r)} h_{t}(x, z) \leq c \inf _{z \in B(y, r)} h_{\theta t}(x, z)
$$

uniformly for $x, y \in \mathcal{X}$, and $r, t>0$ with $r^{m} \leq \nu t$.

We now present the main result of this section. The proof is based on that used by Duong and Robinson in proving [DR, Theorem 3.1]. It relies upon the idea of Hebisch [He] of using $L_{2}$-estimates to obtain weak type $(1,1)$ bounds. Related ideas also appeared earlier in $[\mathrm{F}]$.

Theorem 1. Let $T$ be a bounded linear operator from $L_{2}(\mathcal{X})$ to $L_{2}(\mathcal{X})$ with an associated kernel $k(x, y)$. Assume there exists a class of operators $A_{t}, t>0$, which satisfy the conditions (2) and (3) so that the composite operators $T A_{t}$ have associated kernels $k_{t}(x, y)$ in the sense of (1) and there exist constants $C$ and $c>0$ so that

$$
\int_{d(x, y) \geq c t^{1 / m}}\left|k(x, y)-k_{t}(x, y)\right| d \mu(x) \leq C
$$

for all $y \in \mathcal{X}$.

Then the operator $T$ is of weak type $(1,1)$. Hence, $T$ can be extended from $L_{2}(\mathcal{X}) \cap L_{p}(\mathcal{X})$ to a bounded operator on $L_{p}(\mathcal{X})$ for all $1<p \leq 2$.

Proof. We need to prove that $T$ satisfies weak type $(1,1)$ estimates. Boundedness of $T$ on $L_{p}(\mathcal{X})$ then follows from the Marcinkiewicz interpolation theorem.

Our proof makes use of the Calderón-Zygmund decomposition to decompose an integrable function into "good" and "bad" parts (see, for example, $[\mathrm{CW}])$, then each part is analysed separately.

Given $f \in L_{1}(\mathcal{X}) \cap L_{2}(\mathcal{X})$ and $\alpha>\|f\|_{1}(\mu(\mathcal{X}))^{-1}$, then there exist a constant $c$ independent of $f$ and $\alpha$, and a decomposition

$$
f=g+b=g+\sum_{i} b_{i}
$$


so that

a) $|g(x)| \leq c \alpha$ for almost all $x \in \mathcal{X}$,

b) there exists a sequence of balls $Q_{i}$ so that the support of each $b_{i}$ is contained in $Q_{i}$ and

$$
\int\left|b_{i}(x)\right| d \mu(x) \leq c \alpha \mu\left(Q_{i}\right)
$$

c) $\sum_{i} \mu\left(Q_{i}\right) \leq \frac{c}{\alpha} \int|f(x)| d \mu(x)$,

d) each point of $\mathcal{X}$ is contained in at most a finite number $N$ of the balls $Q_{i}$.

Note that if $\mu(\mathcal{X})=\infty$, then $\|f\|_{1}(\mu(\mathcal{X}))^{-1}$ means 0 . Besides that, the functions $b_{i}$ are usually chosen to satisfy $\int b_{i} d \mu(x)=0$ as well, but we do not need this property.

Conditions b) and c) also imply that $\|b\|_{1} \leq c\|f\|_{1}$ and hence that $\|g\|_{1} \leq(1+c)\|f\|_{1}$.

We have

$$
\begin{aligned}
& \mu(\{x:|T f(x)|>\alpha\}) \\
& \quad \leq \mu\left(\left\{x:|T g(x)|>\frac{\alpha}{2}\right\}\right)+\mu\left(\left\{x:|T b(x)|>\frac{\alpha}{2}\right\}\right) .
\end{aligned}
$$

It is not difficult to check that $g \in L_{2}(\mathcal{X})$. Using the facts that $T$ is bounded on $L_{2}(\mathcal{X})$ and that $|g(x)| \leq c \alpha$, we obtain

$$
\mu\left(\left\{x:|T g(x)|>\frac{\alpha}{2}\right\}\right) \leq 4 \alpha^{-2}\|T g\|_{2}^{2} \leq c_{1} \alpha^{-2}\|g\|_{2}^{2} \leq \frac{c_{2}}{\alpha}\|f\|_{1}
$$

Concerning the "bad" part $b(x)$, we temporarily fix a $b_{i}$ whose support is contained in $Q_{i}$, then choose $t_{i}=r_{i}^{m}$ where $m$ is the constant appearing in (3) and $r_{i}$ is the radius of the ball $Q_{i}$. We then decompose

$$
T b_{i}(x)=T A_{t_{i}} b_{i}(x)+\left(T-T A_{t_{i}}\right) b_{i}(x) .
$$

To analyse $T A_{t_{i}} b_{i}(x)$, we first estimate the function $A_{t_{i}} b_{i}$. Since

$$
A_{t_{i}} b_{i}(x)=\int_{\mathcal{X}} a_{t_{i}}(x, y) b_{i}(y) d \mu(y)
$$


it follows from Lemma 1 that

$$
\begin{aligned}
\left|A_{t_{i}} b_{i}(x)\right| & \leq \int_{\mathcal{X}} h_{t_{i}}(x, y)\left|b_{i}(y)\right| d \mu(y) \\
& \leq\left\|b_{i}\right\|_{1} \sup _{y \in Q_{i}} h_{t_{i}}(x, y) \\
& \leq c \alpha \mu\left(Q_{i}\right) \inf _{y \in Q_{i}} h_{\theta t_{i}}(x, y) \\
& \leq c \alpha \int_{\mathcal{X}} h_{\theta t_{i}}(x, y) \chi_{i}(y) d \mu(y),
\end{aligned}
$$

where $\chi_{i}$ denotes the characteristic function of the ball $Q_{i}$.

Denoting by $M$ the Hardy-Littlewood maximal operator, we then have for any $u \in L_{2}(\mathcal{X})$

$$
\begin{aligned}
\left|\left\langle|u|, A_{t_{i}} b_{i}\right\rangle\right| & \leq c \alpha \int_{\mathcal{X}} \int_{\mathcal{X}}|u(x)| h_{\theta t_{i}}(x, y) \chi_{i}(y) d \mu(y) d \mu(x) \\
& \leq c \alpha\left\langle M|u|, \chi_{i}\right\rangle .
\end{aligned}
$$

Note that the second inequality follows from properties 3 ) and $\left.4^{\prime}\right)$. Since the Hardy-Littlewood maximal operator is bounded on $L_{2}(\mathcal{X})$, (see for example [Ch2]), it follows that

$$
\left\|\sum_{i} A_{t_{i}} b_{i}\right\|_{2} \leq c \alpha\left\|\sum_{i} \chi_{i}\right\|_{2}
$$

We now use properties c) and d) of the Calderón-Zygmund decomposition to obtain the estimate

$$
\left\|\sum_{i} A_{t_{i}} b_{i}\right\|_{2} \leq c \alpha\left(\sum_{i} \mu\left(Q_{i}\right)\right)^{1 / 2} \leq c \alpha^{1 / 2}\|f\|_{1}^{1 / 2} .
$$

Therefore

$$
\begin{aligned}
\mu\left(\left\{x:\left|\sum_{i} T A_{t_{i}} b_{i}(x)\right|>\frac{\alpha}{4}\right\}\right) & \leq 16 \alpha^{-2}\left\|\sum_{i} T A_{t_{i}} b_{i}\right\|_{2}^{2} \\
& \leq c \alpha^{-2}\left\|\sum_{i} A_{t_{i}} b_{i}\right\|_{2}^{2} \\
& \leq \frac{c}{\alpha}\|f\|_{1} .
\end{aligned}
$$


On the other hand

$$
\begin{aligned}
\mu\left(\left\{x: \mid \sum_{i}(T\right.\right. & \left.\left.\left.-T A_{t_{i}}\right) b_{i}(x) \mid>\frac{\alpha}{4}\right\}\right) \\
& \leq \sum_{i} \mu\left(B_{i}\right)+\sum_{i} \frac{4}{\alpha} \int_{{ }^{c} B_{i}}\left|\left(T-T A_{t_{i}}\right) b_{i}(x)\right| d \mu(x)
\end{aligned}
$$

where ${ }^{c} B_{i}$ denotes the complement of $B_{i}$ which is the ball with the same centre $y_{i}$ as that of the ball $Q_{i}$ in the Calderón-Zygmund decomposition but with radius increased by the factor $(1+c)$, where $c$ is the constant in (7). Because of property $\mathrm{c}$ ) of the decomposition and the doubling property of $\mathcal{X}$, we have

$$
\sum_{i} \mu\left(B_{i}\right) \leq c \sum_{i} \mu\left(Q_{i}\right) \leq c \alpha^{-1}\|f\|_{1}
$$

By assumption (7), we have

$$
\begin{aligned}
\int_{{ }^{c} B_{i}} \mid( & \left.-T A_{t_{i}}\right) b_{i}(x) \mid d \mu(x) \\
\leq & \int_{{ }^{c} B_{i}}\left|\int_{\mathcal{X}} k(x, y)-k_{t_{i}}(x, y) b_{i}(y) d \mu(y)\right| d \mu(x) \\
& \leq \int_{\mathcal{X}}\left|b_{i}(y)\right|\left(\int_{d(x, y) \geq c t_{i}^{1 / m}}\left|k(x, y)-k_{t_{i}}(x, y)\right| d \mu(x)\right) d \mu(y) \\
& \leq C\left\|b_{i}\right\|_{1}
\end{aligned}
$$

because $B\left(y ; c t_{i}^{1 / m}\right) \subset B_{i}$.

Therefore

$$
\sum_{i} \frac{1}{\alpha} \int_{{ }^{B} B_{i}}\left|\left(T-T A_{t_{i}}\right) b_{i}(x)\right| d \mu(x) \leq \sum_{i} \frac{C}{\alpha}\left\|b_{i}\right\|_{1} \leq \frac{C\|f\|_{1}}{\alpha}
$$

Combining the estimates (8), (11), (12) and (13), the theorem is proved.

REMARK.

i) It is straightforward from the proof of Theorem 1, that the existence of both the kernels $k(x, y)$ of $T$ and $k_{t}(x, y)$ of $T A_{t}$ is not necessary. We only need to assume that the difference operator $T-T A_{t}$ has 
an associated kernel so that this kernel (in place of $k(x, y)-k_{t}(x, y)$ ) satisfies Condition 7. This remark also applies to Theorem 2.

ii) In Theorem 1, the assumption on boundedness of $T$ on the space $L_{2}(\mathcal{X})$ can be replaced by boundedness of $T$ on a space $L_{p_{o}}(\mathcal{X})$ for some $p_{0}>1$. The proof would need only minor changes to show that $T$ is of weak type $(1,1)$, hence bounded on $L_{p}(\mathcal{X})$ for all $1<p \leq p_{o}$.

iii) Theorem 1 and a standard duality argument give the following result.

Let $T$ be a bounded linear operator from $L_{2}(\mathcal{X})$ into $L_{2}(\mathcal{X})$ with an associated kernel $k(x, y)$ in the sense of (1). Assume there exists a class of operators $B_{t}$ whose kernels satisfy the conditions (2) and (3) so that the composite operators $B_{t} T$ have associated kernels $K_{t}(x, y)$ in the sense of (1), and there exist constants $c>0$ and $C$ so that

$$
\int_{d(x, y) \geq c t^{1 / m}}\left|k(x, y)-K_{t}(x, y)\right| d \mu(y) \leq C,
$$

for all $x \in \mathcal{X}$.

Then the adjoint operator $T^{*}$ is of weak type $(1,1)$. Hence, $T$ can be extended from $L_{2}(\mathcal{X}) \cap L_{p}(\mathcal{X})$ to a bounded operator on $L_{p}(\mathcal{X})$ for all $2 \leq p<\infty$.

Natural questions about Theorem 1 are how strong is the assumption (7), and what is its relation with the Hörmander integral condition. We shall show that, for suitably chosen $A_{t}$, our condition (7) is actually a consequence of the Hörmander integral condition for spaces of homogeneous type.

Proposition 1. Assume that $T$ has an associated kernel $k(x, y)$ which satisfies the Hörmander integral condition, i.e. there exist constants $C$ and $\delta>1$, so that

$$
\int_{d(x, y) \geq \delta d(y, z)}|k(x, y)-k(x, z)| d \mu(x) \leq C
$$

for all $y, z \in \mathcal{X}$. Let $A_{t}$ be approximations to the identity which are represented by kernels $a_{t}(x, y)$ satisfying conditions (2), (3), (5) and (6). 
Then the kernels $k_{t}(x, y)$ of $T A_{t}$ satisfy condition (7) of Theorem 1. More precisely, there exist constants $c$ and $\beta$ so that

$$
\int_{d(x, y) \geq \beta t^{1 / m}}\left|k(x, y)-k_{t}(x, y)\right| d \mu(x) \leq c,
$$

for all $y \in \mathcal{X}$.

Proof. Choose $\delta>1$ and let $\beta=c_{0} \delta$ where $c_{0}$ is the constant so that $a_{t}(x, y)=0$ when $d(x, y) \geq c_{0} t^{1 / m}$. Then, for $x, y \in \mathcal{X}$ so that $d(x, y) \geq \beta t^{1 / m}$,

$$
k_{t}(x, y)=\int_{\mathcal{X}} k(x, z) a_{t}(z, y) d \mu(z) .
$$

For all $y \in \mathcal{X}$

$$
\begin{aligned}
& \int_{d(x, y) \geq \beta t^{1 / m}}\left|k(x, y)-k_{t}(x, y)\right| d \mu(x) \\
& =\int_{d(x, y) \geq \beta t^{1 / m}}\left|k(x, y)-\int_{\mathcal{X}} k(x, z) a_{t}(z, y) d \mu(z)\right| d \mu(x) \\
& =\int_{d(x, y) \geq \beta t^{1 / m}} a_{t}(z, y) d \mu(z) \\
& \cdot\left|k(x, y) \int_{d(z, y) \leq c_{0} t^{1 / m}}-\int_{d(z, y) \leq c_{0} t^{1 / m}} k(x, z) a_{t}(z, y) d \mu(z)\right| d \mu(x) \\
& \leq \sup _{d(z, y) \leq c_{0} t^{1 / m}}\left(\int_{d(x, y) \geq \beta t^{1 / m}}|k(x, y)-k(x, z)| d \mu(x)\right) \\
& \quad \cdot\left(\int_{d(z, y) \leq c_{0} t^{1 / m}}\left|a_{t}(z, y)\right| d \mu(z)\right) \\
& \leq c_{1} \sup _{d(z, y) \leq c_{0} t^{1 / m}}\left(\int_{d(x, y) \geq \delta c_{0} t^{1 / m}}|k(x, y)-k(x, z)| d \mu(x)\right) \\
& \leq c .
\end{aligned}
$$

Note that the second equality follows from property (6), the second inequality is using the estimate

$$
\int_{\mathcal{X}}\left|a_{t}(z, y)\right| d \mu(z) \leq \int_{\mathcal{X}} h_{t}(z, y) d \mu(z) \leq c_{1},
$$


and the last inequality follows from the Hörmander integral condition.

\section{Singular integral operators on measurable subsets of a space of homogeneous type.}

We assume in this section that $\Omega$ is a measurable subset of a space of homogeneous type $(\mathcal{X}, d, \mu)$. An example of $\Omega$ is an open domain of the Euclidean space $\mathbb{R}^{n}$. If $\Omega$ possesses certain smoothness on its boundary, for example Lipschitz boundary, then it is a space of homogeneous type and results of Section 2 are applicable. However, a general measurable set $\Omega$ needs not satisfy the doubling property, hence it is not a space of homogeneous type. Such a measurable set $\Omega$ appears naturally in boundary value problems, for example partial differential equations with Dirichlet boundary conditions.

Given a bounded linear operator $T$ on $L_{2}(\Omega)$ with an associated kernel $k(x, y)$, the question is to find a sufficient condition on $k(x, y)$ for $T$ to be of weak type $(1,1)$. The main problem in this case is the fact that the Calderón-Zygmund theory is not directly applicable. For example, the Calderón-Zygmund decomposition is not valid on $\Omega$, nor is the Hardy-Littlewood maximal operator bounded, as was needed in proving the estimate (9).

A key observation to solve this problem is surprisingly simple. Given a linear operator $T$ which maps $L_{p}(\Omega)$ into itself for some $p$, define an associated operator $\widetilde{T}$ on $L_{p}(\mathcal{X})$ by

$$
\widetilde{T}(u)(x)= \begin{cases}T\left(\chi_{\Omega} u\right)(x), & x \in \Omega, \\ 0, & x \notin \Omega,\end{cases}
$$

where $\chi_{\Omega}$ is the characteristic function on $\Omega$. Then $T$ is bounded on $L_{p}(\Omega)$ if and only if $\widetilde{T}$ is bounded on $L_{p}(\mathcal{X})$, also $T$ is of weak type $(1,1)$ on $\Omega$ if and only if $\widetilde{T}$ is of weak type $(1,1)$ on $\mathcal{X}$.

It is straightforward to check the above equivalences, so we leave them to reader. Note that if $T$ has an associated kernel $k(x, y)$ in the sense of (1), then $\widetilde{T}$ also has an associated kernel $\tilde{k}(x, y)$ in the sense of (1), given by

$$
\tilde{k}(x, y)= \begin{cases}k(x, y), & \text { when } x \in \Omega \text { and } y \in \Omega, \\ 0, & \text { otherwise }\end{cases}
$$

We can see immediately that the condition that the kernel $k(x, y)$ of $T$ satisfies the Hörmander condition is not sufficient for the kernel $k(x, y)$ 
of $\widetilde{T}$ to satisfy the Hörmander condition. By using $\widetilde{T}$, what we do is to transform the question of boundedness of $T$ on a measurable set $\Omega$ to the boundedness of $\widetilde{T}$ on a better space (of homogeneous type) $\mathcal{X}$, but the kernel of $\widetilde{T}$ could be discontinuous. However, the proof of Theorem 1 makes use of the upper bounds on $a_{t}(x, y)$ and condition (7), and does not require any continuity assumptions on $k(x, y)$.

From now on, to differentiate between a ball in $\mathcal{X}$ and a ball in $\Omega$, we use the notations $B^{\mathcal{X}}$ and $B^{\Omega}$.

The main theorem of this section is the following.

Theorem 2. Let $T$ be a bounded linear operator from $L_{2}(\Omega)$ to $L_{2}(\Omega)$ with an associated kernel $k(x, y)$ in the sense of (1). Assume there exists a class of operators $A_{t}, t>0$, with kernels $a_{t}(x, y)$ defined on $L_{2}(\Omega)$ so that:

a)

$$
A_{t} u(x)=\int_{\mathcal{X}} a_{t}(x, y) u(y) d \mu(y),
$$

for any function $u \in L_{2}(\Omega) \cap L_{1}(\Omega)$, and the kernels $a_{t}(x, y)$ satisfy the following conditions

$$
\left|a_{t}(x, y)\right| \leq h_{t}(x, y)
$$

for all $x, y \in \Omega$, where $h_{t}(x, y)$ is defined on $\mathcal{X} \times \mathcal{X}$ by

$$
h_{t}(x, y)=\left(\mu\left(B^{\mathcal{X}}\left(x ; t^{1 / m}\right)\right)\right)^{-1} s\left(d(x, y)^{m} t^{-1}\right),
$$

and $s$ is a positive, bounded, decreasing function satisfying

$$
\lim _{r \rightarrow \infty} r^{n+\kappa} s\left(r^{m}\right)=0
$$

for some $\kappa>0$ with $n$ the "dimension" entering the strong homogeneity property of $\mathcal{X}$,

b) the composite operators $T A_{t}$ have associated kernels $k_{t}(x, y)$ in the sense of (1) and there exist constants $C$ and $c>0$ so that

$$
\int_{d(x, y) \geq c t^{1 / m}}\left|k(x, y)-k_{t}(x, y)\right| d \mu(x) \leq C,
$$

for all $y \in \Omega$. 
Then the operator $T$ is of weak type $(1,1)$. Hence, $T$ can be extended from $L_{2}(\Omega) \cap L_{p}(\Omega)$ to a bounded operator on $L_{p}(\Omega)$ for all $1<p \leq 2$.

Proof. First observe that $\widetilde{T A_{t}}=\widetilde{T} \widetilde{A}_{t}$ where $\widetilde{T}, \widetilde{A_{t}}$ and $\widetilde{T A_{t}}$ are defined on $L_{2}(\mathcal{X})$ as described above. Moreover $\widetilde{T}$ and $\widetilde{T} A_{t}$ have kernels $\tilde{k}(x, y)$ and $\tilde{k}_{t}(x, y)$ in the sense of $(1)$, where $\tilde{k}$ was defined above and

$$
\tilde{k}_{t}(x, y)= \begin{cases}k_{t}(x, y), & \text { when } x \in \Omega \text { and } y \in \Omega, \\ 0, & \text { otherwise }\end{cases}
$$

Further, $\widetilde{A}_{t}$ is represented by the kernel

$$
\tilde{a}_{t}(x, y)= \begin{cases}a_{t}(x, y), & \text { when } x \in \Omega \text { and } y \in \Omega, \\ 0, & \text { otherwise, }\end{cases}
$$

which is readily seen to satisfy conditions (2) and (3) on $\mathcal{X} \times \mathcal{X}$.

Conditions (15), (16), and (17) imply that the operator $\widetilde{T}$ satisfies the hypotheses of Theorem 1 , hence it is of weak type $(1,1)$ on $\mathcal{X}$. Therefore, $T$ is of weak type $(1,1)$ on $\Omega$ and Theorem 2 is proved.

REMARK. Assume there exist $B_{t}$ which satisfy (15) and (16) so that the composite operators $B_{t} T$ satisfy property (14). A standard duality argument shows that the adjoint operator of $T$ is bounded on $L_{p}(\Omega)$ for $1<p \leq 2$, hence $T$ is bounded on $L_{p}(\Omega)$ for $2 \leq p<\infty$.

\section{Boundedness of maximal truncated operators on $L_{p}$ spaces.}

\subsection{The case of spaces of homogeneous type.}

In this subsection, we assume that $\mathcal{X}$ is a space of homogeneous type equipped with a metric $d$ and a measure $\mu$. Let $T$ be a bounded operator on $L_{2}(\mathcal{X})$ with an associated kernel $k(x, y)$ in the sense of (1). Our aim is to investigate the maximal truncated operator $T_{*}$ which is defined by

$$
T_{*} f(x)=\sup _{\varepsilon>0}\left|T_{\varepsilon} f(x)\right|,
$$

where $T_{\varepsilon}$ is the truncated singular operator defined by

$$
T_{\varepsilon} f(x)=\int_{d(x, y) \geq \varepsilon} k(x, y) f(y) d \mu(y) .
$$


The main result of this section is the following theorem.

Theorem 3. We assume the following conditions. $k(x, y)$.

a) $T$ is a bounded operator on $L_{2}(\mathcal{X})$ with an associated kernel

b) There exists a class of operators $A_{t}$ which satisfy the conditions (2) and (3) so that the composite operators $T A_{t}$ have associated kernels $k_{t}(x, y)$ in the sense of (1). Also assume that there exist constants $c_{1}$ and $\delta>0$ so that

$$
\int_{d(x, y) \geq \delta t^{1 / m}}\left|k(x, y)-k_{t}(x, y)\right| d \mu(x) \leq c_{1},
$$

for all $y \in \mathcal{X}$.

c) There exists a class of operators $B_{t}$ represented by kernels $b_{t}(x, y)$ which satisfy the upper bounds $h_{t}(x, y)$ defined by (3), and the composite operators $B_{t} T$ have kernels $K_{t}(x, y)$. Also assume that there exist positive constants $\alpha, c_{2}, c_{3}$ and $c_{4}$ so that

$$
\left|K_{t}(x, y)\right| \leq c_{2}\left(\mu\left(B\left(x ; t^{1 / m}\right)\right)\right)^{-1}, \quad \text { when } d(x, y) \leq c_{3} t^{1 / m}
$$

and

$$
\left|K_{t}(x, y)-k(x, y)\right| \leq c_{4}(\mu(B(x ; d(x, y))))^{-1} \frac{t^{\alpha / m}}{d(x, y)^{\alpha}}
$$

when $d(x, y) \geq c_{3} t^{1 / m}$. Then $T_{*}$ is bounded on $L_{p}(\mathcal{X})$ for all $p, 1<$ $p<\infty$.

Proof. It follows from conditions (5) and (19), Theorem 1 and a duality argument that $T$ is bounded on $L_{p}(\mathcal{X})$ for $1<p<\infty$. Without any loss of generality, we prove the theorem with $c_{3}=1$. For a fixed $\varepsilon>0$, we write

$$
T_{\varepsilon} u(x)=B_{\varepsilon^{m}} T u(x)-\left(B_{\varepsilon^{m}} T-T_{\varepsilon}\right) u(x) .
$$

Since the class of operators $B_{t}$ satisfies conditions (2) and (3), we have

$$
\left|B_{\varepsilon^{m}} T u(x)\right| \leq c M(|T u(x)|),
$$


where $M$ is the Hardy-Littlewood maximal operator, and $c$ is a constant independent of $\varepsilon$.

The kernel of the operator $\left(B_{\varepsilon^{m}} T-T_{\varepsilon}\right)$ is given by $\left(K_{\varepsilon^{m}}(x, y)-\right.$ $\left.k_{\varepsilon}(x, y)\right)$ where $k_{\varepsilon}(x, y)=k(x, y)$ if $d(x, y) \geq \varepsilon$ and $k_{\varepsilon}(x, y)=0$ otherwise. There are two cases:

Case 1. $d(x, y)<\varepsilon$, then $k_{\varepsilon}(x, y)=0$ and it follows from (18) that

$$
\left|K_{\varepsilon^{m}}(x, y)-k_{\varepsilon}(x, y)\right|=\left|K_{\varepsilon^{m}}(x, y)\right| \leq c_{2} \frac{1}{\mu(B(x ; \varepsilon))} .
$$

Case 2. $d(x, y) \geq \varepsilon$, then $k_{\varepsilon}(x, y)=k(x, y)$ and it follows from (19) that

$$
\left|K_{\varepsilon^{m}}(x, y)-k_{\varepsilon}(x, y)\right| \leq c_{4} \frac{1}{\mu(B(x ; d(x, y)))}\left(\frac{\varepsilon}{d(x, y)}\right)^{\alpha}
$$

for some $\alpha>0$.

Therefore

$$
\begin{aligned}
\mid\left(B_{\varepsilon^{m}} T-\right. & \left.T_{\varepsilon}\right) u(x) \mid \\
\leq & c \int_{d(x, y) \leq \varepsilon} \frac{1}{\mu(B(x ; \varepsilon))}|u(y)| d \mu(y) \\
& +c \int_{d(x, y)>\varepsilon} \frac{1}{\mu(B(x ; d(x, y)))}\left(\frac{\varepsilon}{d(x, y)}\right)^{\alpha}|u(y)| d \mu(y) \\
\leq & c \frac{1}{\mu(B(x ; \varepsilon))} \int_{d(x, y) \leq \varepsilon}|u(y)| d \mu(y) \\
& +c \sum_{k=1}^{\infty} \frac{1}{2^{k \alpha}} \frac{1}{\mu\left(B\left(x ; 2^{k+1} \varepsilon\right)\right)} \int_{d(x, y) \leq 2^{k+1} \varepsilon}|u(y)| d \mu(y) \\
\leq & c M(|u|(x)),
\end{aligned}
$$

where again $M$ is the Hardy-Littlewood maximal operator, and $c$ is a constant independent of $\varepsilon$. Therefore

$$
\sup _{\varepsilon>0}\left|\left(B_{\varepsilon^{m}} T-T_{\varepsilon}\right) u(x)\right| \leq c M(|u|(x)) .
$$

Combining estimates (20), (21) with boundedness of $T$ and the HardyLittlewood maximal operator on $L_{p}(\mathcal{X})$, we obtain boundedness of $T_{*}$ on $L_{p}(\mathcal{X}), 1<p<\infty$. 
In the next proposition, we show that, for suitably chosen $B_{t}$, our condition (19) is a consequence of the Hölder continuity estimates on the kernel.

Proposition 2. Assume that for some $\alpha>0$ and $c_{1}>1$, the kernel $k(x, y)$ associated with $T$ satisfies the condition

$$
|k(z, y)-k(x, y)| \leq c \frac{1}{\mu(B(x ; d(x, y))}\left(\frac{d(x, z)}{d(x, y)}\right)^{\alpha},
$$

when $d(x, y) \geq c_{1} d(x, z)$. Let $B_{t}$ be approximations to the identity which are represented by kernels $b_{t}(x, y)$ which satisfy (3), (5) and $\int_{\mathcal{X}} b_{t}(x, y) d \mu(y)=1$, for all $x \in \mathcal{X}, t>0$.

Then the kernels $K_{t}(x, y)$ associated with $B_{t} T$ satisfy condition (19), i.e. there exists a constant $c$ so that

$$
\left|K_{t}(x, y)-k(x, y)\right| \leq c\left(\mu(B(x ; d(x, y)))^{-1} \frac{t^{\alpha / m}}{d(x, y)^{\alpha}}\right.
$$

for $d(x, y) \geq c_{0} c_{1} t^{1 / m}$ where $c_{0}$ is the constant appearing in condition $(5)$.

Proof. Suppose that $d(x, y) \geq c_{0} c_{1} t^{1 / m}$. Then

$$
\begin{aligned}
\mid k(x, y)- & K_{t}(x, y) \mid \\
= & \left|k(x, y)-\int_{\mathcal{X}} k(x, z) b_{t}(z, y) d \mu(z)\right| \\
= & \mid k(x, y) \int_{d(z, y) \leq c_{0} t^{1 / m}} b_{t}(z, y) d \mu(z) \\
& -\int_{d(z, y) \leq c_{0} t^{1 / m}} k(x, z) b_{t}(z, y) d \mu(z) \mid \\
\leq & \int_{d(z, y) \leq c_{0} t^{1 / m}}|k(x, y)-k(x, z)|\left|b_{t}(z, y)\right| d \mu(z) \\
\leq & c\left(\mu(B(x ; d(x, y)))^{-1} \frac{t^{\alpha / m}}{d(x, y)^{\alpha}} \int_{d(z, y) \leq c_{0} t^{1 / m}}\left|b_{t}(z, y)\right| d \mu(z)\right. \\
\leq & c\left(\mu(B(x ; d(x, y)))^{-1} \frac{t^{\alpha / m}}{d(x, y)^{\alpha}} .\right.
\end{aligned}
$$


Note that the second equality is using condition (6) and the second inequality follows from (22).

REMARK. Propositions 1 and 2 show that conditions (7) and (19) are weaker than the usual conditions which guarantee $L_{p}$ boundedness of maximal truncated operators. See, for example [St2, Chapter 1]. However, we need the extra assumption (18). In the case of functional calculi of generators of semigroups with suitable heat kernel bounds, condition (18) is satisfied without extra regularity conditions on the kernel of $T$. See Theorem 6 .

\subsection{The case of measurable subsets of a space of homogeneous type.}

We now assume that $\Omega$ is a measurable subset of a space of homogeneous type $(\mathcal{X}, d, \mu)$ as in Section 3 . By strengthening the assumptions on the kernel of $T$ in Theorem 3, we can obtain boundedness of maximal truncated operators on $L_{p}$ spaces as follows.

Theorem 4. Let $T$ be a bounded operator on $L_{2}(\Omega)$ with an associated kernel $k(x, y)$. We assume the following conditions.

a) There exists a class of operators $A_{t}, t>0$, represented by kernels $a_{t}(x, y)$ which satisfy conditions (15) and (16) so that the composite operators $T A_{t}$ have associated kernels $k_{t}(x, y)$ in the sense of (1), and there exist constants $c$ and $c_{1}$ so that

$$
\int_{d(x, y) \geq c t^{1 / m}}\left|k(x, y)-k_{t}(x, y)\right| d \mu(x) \leq c_{1},
$$

for all $y \in \Omega$.

b) There exists a class of operators $B_{t}, t>0$, represented by kernels $b_{t}(x, y)$ which satisfy conditions (15) and (16) so that the composite operators $B_{t} T$ have kernels $K_{t}(x, y)$, and $K_{t}(x, y)$ satisfy the following conditions

$$
\left|K_{t}(x, y)\right| \leq c\left(\mu\left(B^{\mathcal{X}}\left(x ; t^{1 / m}\right)\right)\right)^{-1},
$$

for all $x, y \in \Omega$ such that $d(x, y) \leq c_{2} t^{1 / m}$,

$$
\left|K_{t}(x, y)-k(x, y)\right| \leq c\left(\mu\left(B^{\mathcal{X}}(x ; d(x, y))\right)^{-1} \frac{t^{\alpha / m}}{d(x, y)^{\alpha}}\right.
$$


for all $x, y \in \Omega$ such that $d(x, y) \geq c_{2} t^{1 / m}$.

Then $T_{*}$ is bounded on $L_{p}(\Omega)$ for all $p, 1<p<\infty$.

Proof. There is no loss of generality in proving the theorem with $c_{2}=1$.

It follows from Theorem 2 and a duality argument that $T$ is bounded on $L_{p}(\Omega)$ for $1<p<\infty$.

Given a function $u \in L_{1}(\Omega) \cap L_{2}(\Omega)$ and $\varepsilon>0$, write

$$
T_{\varepsilon} u(x)=B_{\varepsilon^{m}} T u(x)+\left(B_{\varepsilon^{m}} T-T_{\varepsilon}\right) u(x) .
$$

Consider the term $B_{\varepsilon} T u(x)$. Let $v$ be the extension of $T u$ from $\Omega$ to $\mathcal{X}$ by putting $v(x)=0$ for $x \notin \Omega$, then $\|T u\|_{L_{p}(\Omega)}=\|v\|_{\mathcal{L}_{p}(\mathcal{X})}$ for $1 \leq p \leq \infty$. Similarly, let $w_{\varepsilon}$ be the extension of $B_{\varepsilon^{m}} T u$ from $\Omega$ to $\mathcal{X}$ by putting $w(x)=0$ for $x \notin \Omega$. Since

$$
B_{\varepsilon^{m}} T u(x)=\int_{\mathcal{X}} b_{\varepsilon^{m}}(x, y) T u(y) d \mu(y)
$$

and the kernels $b_{t}(x, y)$ of $B_{t}(x, y)$ satisfy (15) and (16), we have for $x \in \mathcal{X}$,

$$
\left|w_{\varepsilon}(x)\right| \leq c M(|v|(x)),
$$

where $M$ is the Hardy-Littlewood maximal operator, and $c$ is a constant independent of $\varepsilon$. This gives

$$
\sup _{\varepsilon>0}\left|w_{\varepsilon}(x)\right| \leq c M(|v|(x)) \text {. }
$$

The next step is to extend $u$ to $\mathcal{X}$ by putting $u(x)=0$ for $x \notin \Omega$, and denote the extension by $u^{\mathcal{X}}$. Then $\|u\|_{L_{p}(\Omega)}=\left\|u^{\mathcal{X}}\right\|_{L_{p}(\mathcal{X})}$. It then follows from assumption b) and the argument of (21) that

$$
\sup _{\varepsilon>0}\left|\left(B_{\varepsilon^{m}} T-T_{\varepsilon}\right) u(x)\right| \leq c M\left(\left|u^{\mathcal{X}}\right|(x)\right) .
$$

Estimates (26) and (27) imply the boundedness of $T_{*}$ on $L_{p}(\Omega)$ for all $p, 1<p<\infty$.

A consequence of the boundedness of the maximal truncated operator $T_{*}$ is pointwise almost everywhere convergence of the limit

$$
\lim _{\varepsilon \rightarrow 0} T_{\varepsilon} u(x)
$$


More precisely, we have the following lemma.

Lemma 2. Assume that the operator T satisfies the conditions of Theorem 3. Let $1<p<\infty$, and assume that $\lim _{\varepsilon \rightarrow 0} T_{\varepsilon} u(x)$ exists almost everywhere for every $u$ in a dense subspace of $L_{p}(\Omega)$, then $\lim _{\varepsilon \rightarrow 0} T_{\varepsilon} u(x)$ exists almost everywhere for every function $u \in L_{p}(\Omega)$.

Proof. Lemma 2 follows from a standard argument of proving the existence of almost everywhere pointwise limits as a consequence of the corresponding maximal inequality. See, for example [St1, p. 45].

\section{Applications: Riesz transforms and holomorphic functional calculi of elliptic operators.}

\subsection{Definitions.}

We first give some preliminary definitions. References are $\left[\mathrm{M}^{\mathrm{c}}\right]$, [CDMcY], [ADMc].

For $0 \leq \omega<\nu<\pi$, define the closed sector in the complex plane $\mathbb{C}$

$$
S_{\omega}=\{\zeta \in \mathbb{C}:|\arg \zeta| \leq \omega\} \cup\{0\}
$$

and denote its interior by $S_{\omega}^{0}$.

We employ the following subspaces of the space $H\left(S_{\nu}^{0}\right)$ of all holomorphic functions on $S_{\nu}^{0}$.

$$
H_{\infty}\left(S_{\nu}^{0}\right)=\left\{f \in H\left(S_{\nu}^{0}\right):\|f\|_{\infty}<\infty\right\},
$$

where $\|f\|_{\infty}=\sup \left\{|f(\zeta)|: \zeta \in S_{\nu}^{0}\right\}$,

$$
\Psi\left(S_{\nu}^{0}\right)=\left\{\psi \in H\left(S_{\nu}^{0}\right): \text { exists } s>0,|\psi(\zeta)| \leq C|\zeta|^{s}\left(1+|\zeta|^{2 s}\right)^{-1}\right\}
$$

and

$$
F\left(S_{\nu}^{0}\right)=\left\{f \in H\left(S_{\nu}^{0}\right): \text { exists } s>0,|f(\zeta)| \leq C\left(|\zeta|^{-s}+|\zeta|^{s}\right)\right\}
$$

so that

$$
\Psi\left(S_{\nu}^{0}\right) \subset H_{\infty}\left(S_{\nu}^{0}\right) \subset F\left(S_{\nu}^{0}\right)
$$

Let $0 \leq \omega<\pi$. A closed operator $L$ in $L_{p}(\mathcal{X})$ is said to be of type $\omega$ if $\sigma(L) \subset S_{\omega}$ and, for each $\nu>\omega$, there exists $C_{\nu}$ such that

$$
\left\|(L-\zeta I)^{-1}\right\| \leq C_{\nu}|\zeta|^{-1}, \quad \zeta \notin S_{\nu} .
$$


By the Hille-Yoshida Theorem, an operator $L$ of type $\omega$ with $\omega<\pi / 2$ is the generator of a bounded holomorphic semigroup $e^{-z L}$ on the sector $S_{\nu}^{0}$ with $\nu=\pi / 2-\omega$.

Suppose that $L$ is a one--one operator of type $\omega$ with dense domain and dense range in $L_{p}(\mathcal{X})$. We can define a functional calculus of $L$ as follows.

If $\psi \in \Psi\left(S_{\nu}^{0}\right)$, then

$$
\psi(L)=\frac{1}{2 \pi i} \int_{\gamma}(L-\zeta I)^{-1} \psi(\zeta) d \zeta,
$$

where $\gamma$ is the contour $\left\{\zeta=r e^{ \pm i \theta}: r \geq 0\right\}$ parametrised clockwise around $S_{\omega}$, and $\omega<\theta<\nu$. Clearly, this integral is absolutely convergent in $\mathcal{L}(\mathcal{X})$, and it is straightforward to show, using Cauchy's theorem, that the definition is independent of the choice of $\theta \in(\omega, \nu)$.

Let $f \in F\left(S_{\nu}^{0}\right)$, so that for some $c$ and $k,|f(\zeta)| \leq c\left(|\zeta|^{k}+|\zeta|^{-k}\right)$ for every $\zeta \in S_{\nu}^{0}$. Let

$$
\psi(\zeta)=\left(\frac{\zeta}{(1+\zeta)^{2}}\right)^{k+1}
$$

Then $\psi, f \psi \in \Psi\left(S_{\nu}^{0}\right)$ and $\psi(L)$ is one-one. So $(f \psi)(L)$ is a bounded operator on $\mathcal{X}$, and $\psi(L)^{-1}$ is a closed operator in $\mathcal{X}$. Define $f(L)$ by

$$
f(L)=(\psi(L))^{-1}(f \psi)(L) .
$$

An important feature of this functional calculus is the following Convergence Lemma.

Convergence Lemma. Let $0 \leq \omega<\nu \leq \pi$. Let $L$ be an operator of type $\omega$ which is one-one with dense domain and range. Let $\left\{f_{\alpha}\right\}$ be a uniformly bounded net in $H_{\infty}\left(S_{\nu}^{0}\right)$, which converges to $f \in H_{\infty}\left(S_{\nu}^{0}\right)$ uniformly on compact subsets of $S_{\nu}^{0}$, such that $\left\{f_{\alpha}(L)\right\}$ is a uniformly bounded net in $\mathcal{L}(\mathcal{X})$. Then $f(L) \in \mathcal{L}(\mathcal{X}), f_{\alpha}(L) u \longrightarrow f(L) u$ for all $u \in \mathcal{X}$, and $\|f(L)\| \leq \sup _{\alpha}\left\|f_{\alpha}(L)\right\|$.

For the proof of the Convergence Lemma, see $\left[\mathrm{M}^{\mathrm{c}}\right]$, or $\left[\mathrm{ADM}^{\mathrm{c}}\right]$. 


\section{2. $L_{p}$ boundedness of Riesz Transforms.}

In this subsection, we assume that $\Omega$ is a measurable subset of a space of homogeneous type $(\mathcal{X}, d, \mu)$ in Section 3 . Let $L$ be a linear operator of type $\omega$ on $L_{2}(\Omega)$ with $\omega<\pi / 2$, so that $(-L)$ generates a holomorphic semigroup $e^{-z L}, 0 \leq|\operatorname{Arg}(z)|<\theta, \theta=\pi / 2-\omega$. Assume that for real $t>0$, the distribution kernels $a_{t}(x, y)$ of $e^{-t L}$ belong to $L_{\infty}(\Omega \times \Omega)$ and satisfy the estimate

$$
\left|a_{t}(x, y)\right| \leq h_{t}(x, y)
$$

for $x, y \in \Omega$ where $h_{t}$ is defined on $\mathcal{X} \times \mathcal{X}$ by (3) and.

For $0 \leq \alpha<1, \nu>0$, define $F_{\alpha}\left(S_{\nu}^{0}\right)$ as follows

$$
F_{\alpha}\left(S_{\nu}^{0}\right)=\left\{f \in H\left(S_{\nu}^{0}\right): \text { exists } c,|f(\zeta)| \leq C|\zeta|^{-\alpha}\right\} .
$$

Assume that $g \in F_{\alpha}\left(S_{\nu}^{0}\right)$ for some $\nu>\pi / 2$, and that $D$ is a densely defined linear operator on $L_{2}(\Omega)$ which possesses the following two properties:

a) $D g(L)$ is bounded on $L_{2}(\Omega)$,

b) the function $D a_{t}, t>0$, obtained by the action of $D$ on $a_{t}(x, y)$ with respect to the variable $x$, satisfies the estimate

$$
\left|D a_{t}(x, y)\right| \leq c t^{-\alpha} h_{t}(x, y)
$$

for all $x, y \in \Omega$.

The main result of this section is the following theorem.

Theorem 5. Under the above assumptions a) and $\mathrm{b}$ ), the operator $D g(L)$ is of weak type $(1,1)$, hence it can be extended to a bounded operator on $L_{p}(\Omega)$ for $1<p \leq 2$.

Before giving the proof of the theorem, we give examples which are applications of our results. Some specific operators which satisfy the assumptions of Theorem 5 are as follows.

i) Let $\mathfrak{g}$ be a finite dimensional nilpotent Lie algebra. Assume that

$$
\mathfrak{g}=\bigoplus_{i=1}^{m} \mathfrak{g}_{i}
$$


as a vector space, where $\left[\mathfrak{g}_{i}, \mathfrak{g}_{j}\right] \subseteq \mathfrak{g}_{i+j}$ for all $i, j$, and $\mathfrak{g}_{1}$ generates $\mathfrak{g}$ as a Lie algebra.

Let $G$ be the associated connected, simply connected Lie group. Then $G$ has homogeneous dimension $d$ given by the formula

$$
d=\sum_{j=1}^{m} j \operatorname{dim}\left(\mathfrak{g}_{j}\right),
$$

where $\operatorname{dim}\left(\mathfrak{g}_{j}\right)$ denotes the dimension of $\mathfrak{g}_{j}$.

Consider any finite basis $\left\{X_{k}\right\}$ of $\mathfrak{g}_{1}$. Each $X_{k}$ can be identified with a unique left invariant vector field on $G$. Define

$$
L=-\sum_{k} X_{k}^{2}
$$

Then the sub-Laplacian $L$ is a left invariant second order differential operator, which is a non-negative self-adjoint operator in $L_{2}(G)$. The Banach spaces $L_{p}(G)$ are defined with respect to Haar measure.

Note that $G$ is a Lie group of polynomial growth, hence it is a space of homogeneous type. Consider the Riesz transforms $X_{k} L^{-1 / 2}$ which are special cases of our operator $D g(L)$ when $D=X_{k}$ and $g(L)=L^{-1 / 2}$. It is not difficult to check that $X_{k} L^{-1 / 2}$ is bounded on $L_{2}(G)$. Gaussian upper bounds on heat kernels and their space derivatives are well known (see, for example [SC]), hence our condition b) is satisfied with $\alpha=1 / 2$. It follows that the Riesz transforms $X_{k} L^{-1 / 2}$ are bounded on $L_{p}(G)$ for $1<p \leq 2$ and are of weak type $(1,1)$. Thus we have simplified the proof of the $L_{p}$ boundedness of the Riesz transforms given by Saloff-Coste [SC], because we have not used the smoothness of the heat kernels in the variable $y$.

In the same setting of $G$, we can also consider the case when $L$ is a $2 m$-th order strongly elliptic operator with constant coefficients (plus a sufficiently large constant $c_{0}$ ), and $D=X_{i_{1}} X_{i_{2}} \cdots X_{i_{n}}$ for some $n<2 m$. Then the operator $D L^{-n / 2 m}$ is bounded on $L_{2}(G)$. The condition (b) is also satisfied with $\alpha=n / 2 m$. See, for example, $[\mathrm{R}$, Chapter 4]. Our theorem then shows that $D L^{-n / 2 m}$ can be extended to a bounded operator on each $L_{p}(G)$ for $1<p \leq 2$.

ii) Let $\mathcal{X}$ be a complete Riemannian manifold with non-negative Ricci curvature, $L$ the Laplace-Beltrami operator, and $D$ a vector field. Then the Riesz transform $D L^{-1 / 2}$ is bounded on $L_{2}(\mathcal{X})$. Upper bounds on the heat kernels and their derivatives can be found, for example, in 
[CLY], [Da2]. Thus the assumptions of Theorem 5 are satisfied (with $\alpha=1 / 2)$, so $D L^{-1 / 2}$ can be extended to a bounded operator on each $L_{p}(\mathcal{X})$ for $1<p \leq 2$.

We now proceed to prove Theorem 5. The following off-diagonal estimate is proved in [DR, Proposition 2.1].

Lemma 3. Let $h_{t}(x, y)$ be given by (3), then for each $\delta, 0<\delta<\kappa-N *$, where $\kappa, N *$ are the constants in (4), there exists $c>0$ so that

$$
\int_{d(x, y) \geq r} h_{t}(x, y) d \mu(x) \leq c\left(1+r^{m} t^{-1}\right)^{-\delta},
$$

uniformly for all $r \geq 0, t>0$ and $y \in \mathcal{X}$.

Proof of TheOrem 5. Observe that, for each positive integer $k$, the powers of the resolvent $(L-\lambda I)^{-k}$ are given by

$$
(L-\lambda I)^{-k}=c_{k} \int_{0}^{\infty} t^{k-1} e^{\lambda t} e^{-t L} d t
$$

when $\lambda<0$. Therefore, the operators $(L-\lambda I)^{-k}$ are represented by kernels $g_{\lambda}^{k}(x, y)$ where

$$
g_{\lambda}^{k}(x, y)=c_{k} \int_{0}^{\infty} t^{k-1} e^{\lambda t} a_{t}(x, y) d t
$$

It follows from this representation and the estimates (3) and (4) on the heat kernels that for a sufficiently large integer $k$, the kernels $g_{\lambda}^{k}(x, y)$ possess upper bounds which are similar to those of the heat kernels. More specifically, there exist $h_{t}(x, y)$ satisfying (3) and (4), possibly with a different function $s$, so that

$$
\left|\lambda^{k} g_{\lambda}^{k}(x, y)\right| \leq h_{t}(x, y)
$$

for all $x, y \in \Omega$, where $t=1 /|\lambda|$.

Similarly, we also have the bound

$$
\left|D \lambda^{k} g_{\lambda}^{k}(x, y)\right| \leq c t^{-\alpha} h_{t}(x, y)
$$

for all $x, y \in \Omega$. 
Choose the class of operators $A_{t}=(t L+I)^{-k}$. By Theorem 2, it suffices to show that condition (17) is satisfied. The kernels $(k(x, y)-$ $\left.k_{t}(x, y)\right)$ in condition (17) are associated with operators $D g(L)(I-(t L+$ $\left.I)^{-k}\right)$. Let $g(L)\left(I-(t L-I)^{-k}\right)=f(L)$ where $f(z)=g(z)(1-(t z+$ $\left.1)^{-k}\right)$. Using the upper bounds on $g(z)$, we see that $f$ belongs to the class $\Psi\left(S_{\nu}^{0}\right)$.

We next represent the operator $f(L)$ by using the semigroup $e^{-z L}$. By $(28), f(L)$ (acting on $L_{2}(\mathcal{X})$ ) is given by

$$
f(L)=\frac{1}{2 \pi i} \int_{\gamma}(L-\lambda I)^{-1} f(\lambda) d \lambda,
$$

where the contour $\gamma=\gamma_{+} \cup \gamma_{-}$is given by $\gamma_{+}(t)=t e^{i \nu}$ for $t \geq 0$ and $\gamma_{-}(t)=-t e^{-i \nu}$ for $t \leq 0$, and $\nu>\pi / 2$.

For $\lambda \in \gamma$, substitute

$$
(L-\lambda I)^{-1}=\int_{0}^{\infty} e^{\lambda s} e^{-s L} d s .
$$

Changing the order of integration gives

$$
f(L)=\int_{0}^{\infty} e^{-s L} n(s) d s
$$

where

$$
n(s)=\frac{1}{2 \pi i} \int_{\gamma} e^{\lambda s} f(\lambda) d \lambda .
$$

Consequently, the kernel $k_{f}(x, y)$ of $f(L)$ is given by

$$
k_{f}(x, y)=\int_{0}^{\infty} a_{s}(x, y) n(s) d s .
$$

It follows from the upper bound on $g(z)$ and assumption b) that

$$
\begin{gathered}
\int_{d(x, y) \geq c t^{1 / m}}\left|k(x, y)-k_{t}(x, y)\right| d \mu(x) \\
\leq c \int_{0}^{\infty} s^{-\alpha}\left(\int_{0}^{\infty}\left|\lambda^{-\alpha} e^{s \lambda}\left(1-(t \lambda+1)^{-k}\right)\right|\right. \\
\left.\cdot\left(\int_{d(x, y) \geq c t^{1 / m}} h_{s}(x, y) d \mu(x)\right) d|\lambda|\right) d s \\
\leq c \int_{0}^{\infty} s^{-\alpha}\left(\int_{0}^{\infty}\left|\lambda^{-\alpha} e^{s \lambda}\left(1-(t \lambda+1)^{-k}\right)\right|\left(1+t s^{-1}\right)^{-\delta} d|\lambda|\right) d s,
\end{gathered}
$$


by Lemma 3 . Observe that $\left|1-(t \lambda+1)^{-k}\right| \leq c$ and $\left|1-(t \lambda+1)^{-k}\right| \leq$ ct $|\lambda|$ when $t|\lambda| \leq 1$. We then split the integral on the right hand side into two parts, $I_{1}$ and $I_{2}$, corresponding to integration over $t|\lambda|>1$ and $t|\lambda| \leq 1$. Then

$$
I_{1} \leq \int_{0}^{\infty} s^{-\alpha} \int_{1 / t}^{\infty} v^{-\alpha} e^{-\beta s v}\left(1+t s^{-1}\right)^{-\delta} d v d s
$$

with $\beta>0$. Changing variables $t v \longrightarrow v$ and $s / t \longrightarrow s$, and choosing a positive $\varepsilon<\delta$, we have

$$
\begin{aligned}
I_{1} \leq & c \int_{0}^{\infty} s^{-\alpha}\left(\int_{1}^{\infty} v^{-\alpha} e^{-\beta s v}\left(1+s^{-1}\right)^{-\delta} d v\right) d s \\
& =c \int_{0}^{\infty} \frac{s^{\delta}}{(1+s)^{\delta}}\left(\int_{1}^{\infty} \frac{1}{s^{1+\varepsilon}} \frac{1}{v^{1+\varepsilon}}(s v)^{1+\varepsilon-\alpha} e^{-\beta s v} d v\right) d s \\
& \leq c \int_{0}^{\infty} \frac{s^{\delta}}{(1+s)^{\delta} s^{1+\varepsilon}}\left(\int_{1}^{\infty} \frac{1}{v^{1+\varepsilon}} d v\right) d s \\
& \quad\left(\text { since }(s v)^{1+\varepsilon-\alpha} e^{-\beta s v}\right. \text { is bounded) } \\
\leq & C .
\end{aligned}
$$

Similarly,

$$
\begin{aligned}
I_{2} & \leq c \int_{0}^{\infty} s^{-\alpha} \int_{0}^{1 / t} v^{-\alpha} t v e^{-\beta s v} d v\left(1+t s^{-1}\right)^{-\delta} d v d s \\
& \leq c \int_{0}^{\infty} s^{-\alpha}\left(1+s^{-1}\right)^{-\delta} \int_{0}^{1} v^{1-\alpha} e^{-\beta s v} d v d s \\
& \leq c \int_{0}^{1} d v \int_{0}^{\infty} w^{-\alpha} e^{-\beta w} d w \\
& \leq C
\end{aligned}
$$

Therefore, condition (17) is satisfied and Theorem 5 follows from Theorem 2.

\section{REMARKS.}

$\alpha$ ) In the assumption $\mathrm{b}$ ), we do not assume any regularity of $a_{t}(x, y)$ in the variable $y$. 
$\beta)$ The theorem is still true for $g \in F_{\alpha}\left(S_{\nu}^{0}\right)$ with $\nu<\pi / 2$ if the upper bounds on $a_{t}(x, y)$ in condition $b$ ) hold for all complex $t \in S_{\theta}^{0}$ with $\theta>\pi / 2-\nu$. This can be achieved by first choosing $\mu=\nu-\varepsilon$, (with $\varepsilon$ to be specified later), using the formula

$$
f(L)=\frac{1}{2 \pi i} \int_{\gamma}(L-\lambda I)^{-1} f(\lambda) d \lambda
$$

where the contour $\gamma=\gamma_{+} \cup \gamma^{-}$is given by $\gamma_{+}(t)=t e^{i \mu}$ for $t \geq 0$ and $\gamma_{-}(t)=-t e^{-i \mu}$ for $t \leq 0$.

We then substitute

$$
(L-\lambda I)^{-1}=\int_{\Gamma} e^{\lambda z} e^{-z L} d z
$$

for $\lambda \in \gamma_{+}$, where $\Gamma$ is given by $\Gamma(t)=t e^{i(\theta-\varepsilon)}$ for $t \geq 0$, and $\varepsilon$ is chosen sufficiently small so that $(\theta+\mu-\varepsilon)>\pi / 2$. We also have similar expression for $\lambda \in \gamma_{-}$. Thus we obtain a similar representation of $f(L)$ to that of (30), and the rest of the proof is the same as before.

$\gamma$ ) The pointwise bound in condition $(b)$ can be replaced by a weaker condition on the $L_{2}$ norm with a suitable weight of $D a_{t}$ (with respect to $x$ variable). See [CD].

\subsection{Holomorphic functional calculi of elliptic operators.}

We again assume that $\Omega$ is a measurable subset of a space of homogeneous type $(\mathcal{X}, d, \mu)$ as in Section 3 .

Let $L$ be a linear operator of type $\omega$ on $L_{2}(\Omega)$ with $\omega<\pi / 2$, hence $L$ generates a holomorphic semigroup $e^{-z L}, 0 \leq|\operatorname{Arg}(z)|<\pi / 2-\omega$.

Theorem 6. Assume the following two conditions.

a) The holomorphic semigroup $e^{-z L},|\operatorname{Arg}(z)|<\pi / 2-\omega$, is represented by kernels $a_{z}(x, y)$ which satisfy, for all $\theta>\omega$, an estimate

$$
\left|a_{z}(x, y)\right| \leq c_{\theta} h_{|z|}(x, y)
$$

for $x, y \in \Omega$ and $|\operatorname{Arg}(z)|<\pi / 2-\theta$, where $h_{t}$ is defined on $\mathcal{X} \times \mathcal{X}$ by $(3)$. 
b) The operator $L$ has a bounded holomorphic functional calculus in $L_{2}(\Omega)$. That is, for any $\nu>\omega$ and $f \in H_{\infty}\left(S_{\nu}^{0}\right)$, the operator $f(L)$ satisfies

$$
\|f(T)\|_{2} \leq c_{\nu}\|f\|_{\infty} .
$$

Then the operator $L$ has a bounded holomorphic functional calculus in $L_{p}(\Omega), 1<p<\infty$, that is,

$$
\|f(L)\|_{p} \leq c_{p, \nu}\|f\|_{\infty}
$$

for all $f \in H_{\infty}\left(S_{\nu}^{0}\right)$.

When $p=1$, the operator $f(L)$ is of weak-type $(1,1)$.

If we denote $T=f(L)$, then the maximal truncated operator $T_{*}$ is bounded on $L_{p}(\Omega)$ for all $p, 1<p<\infty$.

Proof. Given $\pi / 2>\nu>\omega$, choose $\theta$ and $\mu$ such that $\omega<\theta<\mu<\nu$. For $f \in \Psi\left(S_{\nu}^{0}\right)$, represent the operator $f(L)$ by using the semigroup $e^{-z L}$ as before. This gives

$$
f(L)=\int_{\Gamma_{+}} e^{-z L} n_{+}(z) d z+\int_{\Gamma_{-}} e^{-z L} n_{-}(z) d z
$$

where we choose the contour $\Gamma_{+}(s)=s e^{i(\pi / 2-\theta)}$ for $s \geq 0$ and $\Gamma_{-}(s)=$ $-s e^{-i(\pi / 2-\theta)}$ for $s \leq 0$. The functions $n_{ \pm}(z)$ are given by

$$
n_{ \pm}=\frac{1}{2 \pi i} \int_{\gamma_{ \pm}} e^{\lambda z} f(\lambda) d \lambda
$$

where $\gamma_{+}(s)=s e^{i \mu}$ for $t \geq 0$ and $\gamma_{-}(t)=-t e^{-i \mu}$ for $t \leq 0$.

This implies the bound

$$
\left|n_{ \pm}(z)\right| \leq c\|f\|_{\infty}|z|^{-1}
$$

Consequently, the kernel $k_{f}(x, y)$ of $f(L)$ is given by

$$
k_{f}(x, y)=\int_{\Gamma_{+}} a_{z}(x, y) n_{+}(z) d z+\int_{\Gamma_{-}} a_{z}(x, y) n_{-}(z) d z .
$$

Choose operators $A_{t}=e^{-t L}$. Using the upper bounds on the heat kernels and Lemma 3, similar estimates to the terms $I_{1}$ and $I_{2}$ in the proof of Theorem 5 shows that condition (17) of Theorem 2 is satisfied. Therefore, $f(L)$ is bounded on $L_{p}(\Omega)$. The Convergence Lemma then 
allows us to extend $L_{p}$ boundedness of $f(L)$ to all $f \in H_{\infty}\left(S_{\nu}^{0}\right)$, hence the operator $L$ has a bounded holomorphic function calculus in $L_{p}(\Omega)$. Although the extension of the weak type $(1,1)$ estimates from $f(L)$ for $f \in \Psi\left(S_{\nu}^{0}\right)$ to $f(L)$ for $f \in H_{\infty}\left(S_{\nu}^{0}\right)$ does not follow from the Convergence Lemma, it is not difficult. See for example [ADMc ${ }^{\mathrm{c}}$, Lecture 7 , Section N].

To prove the $L_{p}$ boundedness of the maximal truncated operator $T_{*}$, first choose $B_{t}=A_{t}=e^{-t L}$. We then just need to verify conditions (23) and (24) of Theorem 4.

To verify (23), we use the commutative property of functional calculus:

$$
e^{-t L} f(L)=e^{-t L / 2} f(L) e^{-t L / 2} .
$$

Since $e^{-t L}$ maps $L_{1}(\Omega)$ into $L_{1}(\Omega)$ with the operator norm less than a constant, and $e^{-t L}$ maps $L_{1}(\Omega)$ into $L_{\infty}(\Omega)$ with the operator norm less than $\left(\mu\left(B^{\mathcal{X}}\left(x ; t^{-1 / m}\right)\right)\right)^{-1}$, interpolation and duality gives

$$
\begin{aligned}
\left\|e^{-t L / 2}\right\|_{L_{1}(\Omega) \rightarrow L_{2}(\Omega)} & =\left\|e^{-t L / 2}\right\|_{L_{2}(\Omega) \rightarrow L_{\infty}(\Omega)} \\
& \leq c\left(\mu\left(B^{\mathcal{X}}\left(x ; t^{-1 / m}\right)\right)\right)^{-1 / 2} .
\end{aligned}
$$

These estimates, combined with the fact that $f(L)$ is bounded on $L_{2}(\Omega)$, imply condition (23).

The proof of $(24)$ is straightforward. Consider $d(x, y) \geq c t^{1 / m}$, we have

$$
\left|k(x, y)-k_{t}(x, y)\right| \leq c \int_{0}^{\infty}\left|h_{z}(x, y)\right| d|z| \int_{0}^{\infty}\left|\lambda^{-\alpha} e^{z \lambda}\left(1-e^{-t \lambda}\right)\right| d|\lambda| .
$$

Observe that $\left|1-e^{-t \lambda}\right| \leq c$ since $\operatorname{Re}(\lambda) \geq 0$ and $\left|1-e^{-t \lambda}\right| \leq c t|\lambda| \leq$ $c(t|\lambda|)^{\alpha}$ for $0<\alpha<1$ when $t|\lambda| \geq 1$. We then split the integral on the left hand side into two parts, $I_{1}$ and $I_{2}$, corresponding to integration over $t|\lambda|>1$ and $t|\lambda| \leq 1$. Using the heat kernel bounds and elementary integration, similar estimates to those of $I_{1}$ and $I_{2}$ of Theorem 5 show that

$$
\left|K_{t}(x, y)-k(x, y)\right| \leq c\left(\mu\left(B^{\mathcal{X}}(x ; d(x, y))\right)^{-1} \frac{t^{\beta / m}}{d(x, y)^{\beta}},\right.
$$

for some $\beta>0, x, y \in \Omega, d(x, y) \geq t^{1 / m}$. We leave details of these estimates to reader. 


\section{REMARKS.}

a) Condition a) of Theorem 6 can be replaced by a more general condition as follows. Assume that $L$ is an operator of type $\omega$ and that there exists a positive integer $k$ so that the kernels $g_{\lambda}^{k}(x, y)$ of the power of the resolvents $(\lambda L-I)^{-k}$ satisfy the following estimate

$$
\left|g_{\lambda}^{k}(x, y)\right| \leq\left(\mu\left(B^{\mathcal{X}}\left(x ;|\lambda|^{-1 / m}\right)\right)\right)^{-1} s\left(d(x ; y)^{m}|\lambda|\right),
$$

where $s$ is a function which satisfies the decay condition in (3). The proof under this assumption is still the same as that of Theorem 6 , with the operators $(\lambda L-I)^{-k}$ replacing the semigroup $e^{-z L}$. The advantage of this assumption is that the operator $L$ can be of type $\omega$ with $\omega>\pi / 2$, or of type $\omega$ on a double sector.

b) When $\mathcal{X}$ is a space of homogeneous type, the result on boundedness of holomorphic functional calculi of Theorem 6 was first presented in [DR, Theorem 3.1]. Note that the Hörmander integral condition is not applicable when we have no control on smoothness of heat kernels in the space variables.

c) Heat kernel bounds are known for a large class of elliptic and subelliptic operators. Also see $\left[\mathrm{AM}^{\mathrm{c}} \mathrm{T}\right],[\mathrm{A}]$ for recent results on heat kernel bounds for second order elliptic operators with non-smooth coefficients.

d) Theorem 6 gives new results when $\Omega$ is a measurable set with no smoothness on its boundary. An example of an operator on such a domain, which possesses Gaussian bounds on its heat kernels, is the Laplacian on an open subset of Euclidean space $\mathbb{R}^{n}$ subject to Dirichlet boundary conditions. Gaussian upper bounds can be obtained in this case by a simple argument using the comparison principle. More general operators on open domains of $\mathbb{R}^{n}$ which possess Gaussian bounds can be found in [Da1] and $[\mathrm{AE}]$. Indeed Theorem 6 can be applied to prove the general statement of Theorem 5.5 in [AE] on boundedness of holomorphic functional calculi in $L_{p}$ spaces, without the assumption that the boundary has null measure.

Acknowledgements. The authors would like to thank Paul Auscher for helpful suggestions. 


\section{References.}

[ADM $\left.{ }^{\mathrm{C}}\right]$ Albrecht, D., Duong, X., Mc Intosh, A., Operator Theory and Harmonic Analysis. Workshop in Analysis and Geometry. 1995, Proceedings of the Centre for Mathematics and its Applications, ANU 34 (1996), 77-136.

[AE] Arendt, W., ter Elst, A. F. M., Gaussian estimates for second order elliptic operators with boundary conditions. Reports on Applied and Numerical Analysis, Eindhoven University of Technology, 1995.

[A] Auscher, P., Regularity theorems and heat kernels for elliptic operators. J. London Math. Soc. 54 (1996), 284-296.

[AM $\left.{ }^{\mathrm{C}} \mathrm{T}\right]$ Auscher, P., Mc Intosh, A., Tchamitchian, Ph., Heat kernels of second order complex elliptic operators and applications. J. Funct. Anal. 152 (1998), 22-73.

[CLY] Cheng, S. Y., Li, P., Yau, S. T., On the upper estimate of the heat kernel of a complete Riemannian manifold. Amer. J. Math. 103 (1981), 10211063.

[Ch1] Christ, M., Weak-type $(1,1)$ bounds for rough operators. Ann. Math. 128 (1988), 19-42.

[Ch2] Christ, M., Lectures on Singular Integral Operators. CBMS, 1991.

[CR] Christ, M., Rubio de Francia, J. L., Weak-type $(1,1)$ bounds for rough operators II. Invent. Math. 93 (1988), 225-237.

[CW] Coifman, R. R., Weiss, G., Analyse harmonique non-commutative sur certains espaces homogènes. Lecture Notes in Math. Springer-Verlag 242, 1971.

[CD] Coulhon, T., Duong, X. T., Riesz transforms for $1 \leq p \leq 2$. Trans. Amer. Math. Soc. To appear.

[CDM $\left.{ }^{\mathrm{C}} \mathrm{Y}\right]$ Cowling, M., Doust, I., M${ }^{\mathrm{C}}$ Intosh, A., Yagi, A., Banach space operators with a bounded $H^{\infty}$ functional calculus. J. Austral. Math. Soc. Ser. A 60 (1996), 51-89.

[Da1] Davies, E. B., Heat Kernels and Spectral Theory. Cambridge University Press, 1989.

[Da2] Davies, E. B., Pointwise bounds on the space and time derivatives of heat kernels. J. Operator Theory (1989), 367-378.

[Du] Duong, X. T., $H_{\infty}$ functional calculus of elliptic partial differential operators in $L_{p}$ spaces. Ph. D. Thesis. Macquarie University, 1990.

$\left[\mathrm{DM}^{\mathrm{c}}\right]$ Duong, X. T., McIntosh, A., Functional calculi of second order elliptic partial differential operators with bounded measurable coefficients. $J$. Geom. Anal. 6 (1996), 181-205.

[DR] Duong, X. T., Robinson, D., Semigroup kernels, Poisson bounds and holomorphic functional calculus. J. Funct. Anal. 142 (1996), 89-128. 
[F] Fefferman, C., Inequalities for strongly singular convolution operators. Acta Math. 124 (1970), 9-36.

[He] Hebisch, W., A multiplier theorem for Schrödinger operators. Coll. Math. 60 (1990), 659-664.

[Hof] Hofmann, S., Weak $(1,1)$ boundedness of singular integrals with nonsmooth kernel. Proc. Amer. Math. Soc. 103 (1988), 260-264.

[Hör] Hörmander, L., Estimates for translation invariant operators on $L^{p}$ spaces. Acta Math. 104 (1960), 93-139.

[Mc] $\mathrm{M}^{\mathrm{C} I n t o s h}$, A., Operators which have an $H^{\infty}$ functional calculus. Miniconference on Operator Theory and Partial Differential Equations. Proceedings of the Centre for Mathematical Analysis, ANU 14 (1986), 210231.

[R] Robinson, D. W., Elliptic Operators and Lie Groups. Oxford University, 1991.

[SC] Saloff-Coste, L., Analyse sur les groupes de Lie à croissance polynomiale. Arkiv. Mat. 28 (1990), 315-331.

[Se] Seeger, A., Singular integral operators with rough convolution kernels. J. Amer. Math. Soc. 9 (1996), 95-105.

[St1] Stein, E. M., Singular Integrals and Differentiability Properties of Functions. Princeton University Press, 1970.

[St2] Stein, E. M., Harmonic Analysis. Princeton University, 1993.

Recibido: 1 de octubre de 1.996

Xuan Thinh Duong and Alan McIntosh School of Mathematics, Physics, Computing and Electronics

Macquarie University

N.S.W. 2109, AUSTRALIA duong@mpce.mq . edu . au alan@mpce.mq. edu . au

The authors were supported by the Australian Research Council as well as by Macquarie University. 